\section{$\triangle 103$}

Vitamin E Nutritional Status in the Neonate - Evaluation of $\alpha$-Tocopherol Blood Cells and Buccal Mucosal Cells

Daisy E. Kaempf, Tohru Ogihara, Masayuki Miki and Makoto Mino

The aim of this study was an evaluation of vitamin $E$ nutritional status in neonates by determination of $\alpha$-tocopherol in plasma (PL), red blood cells (RBC), platelets (PLT), monocytes (MN), polymorphunclear leucoytes (PMN) and buccal mucosal cells (BMC). Neonates and children up to 16 years of age were evaluated.

Patients, Methods: 65 children and 81 neonates were enrolled in this study. $\alpha$ tocopherol was measured by HPLC-technique with electrochemical detector. Results: (a:mean $\pm S D, n=$ number of patients)

\begin{tabular}{lll}
\hline & Neonates & Children \\
\hline PL $\mu \mathrm{g} / \mathrm{dl}$ & $402 \pm 155^{\mathrm{a}}(8 \mathrm{l})^{\mathrm{n}}$ & $769 \pm 99(65)$ \\
$\mathrm{RBC}, \mu \mathrm{g} / \mathrm{dl}$ packed cells & $203 \pm 48(29)$ & $241 \pm 47(55)$ \\
$\mathrm{PLT}, \mu \mathrm{g} / \mathrm{mg}$ protein & $0,03 \pm 0,02(29)$ & $0,12 \pm 0,05(52)$ \\
$\mathrm{MN}, \mu \mathrm{g} / 10^{9}$ cells & $4,06 \pm 1,02(40)$ & $8,47 \pm 2,66(51)$ \\
$\mathrm{PMN}, \mu \mathrm{g} / 10^{9}$ cells & $2,59 \pm 1,33(24)$ & $4,35 \pm 1,23(25)$ \\
$\mathrm{BMC}, \mathrm{ng} / \mathrm{mg}$ protein & $25,4 \pm 7,6(25)$ & $47,8 \pm 15,8(30)$
\end{tabular}

Conclusion: During the neonatal period, the lowest levels were documented in all blood fractions excluding RI3C, $\alpha$-tocopherol in PL, PLT, MN, PMN and $\mathrm{BMC}$ in newborns amounted to only about one-half or one-third of older child values, implicating a relative vitamin $E$ deficiency in the newborn.

Osaka Medical Collegc. Japan/Heidelberg University Childrens'.Hospital

\section{$\triangle 104$}

NEONATAL SCREENING FOR GLUCOSE-6-PHOSPHATE DEHYDROGENASE (G-6-PD) DEFICIENCY: Sex Distribution and Role of Paternity Michael Kaplan, Cathy Hammerman, Ayala Abramov. Depts of Neonatology and Pediatrics, Shaare Zedek Med Ctr, Hebrew University, Jerusalem, Israel.

G-6-PD deficiency is a common condition associated with neonatal jaundice and even kernicterus. As it is an X-linked condition, many population surveys and studies of neonatal jaundice have been confined to males. Classically, both parents should be circumstances the mother only need be to be similarly affected; however, under certain infants of high risk mothers in our Sephardic Jewish population to determine the prevalence of the condition in both sexes, and determined the marriage patterns of the parents of the female enzyme deficient infants. A qualitative color reduction screening method was used (Sigma) capable of differentiating between those normal for G-6-PD or grossly deficient in the enzyme. A total of 806 infants was screened between 1989 and 1992: 127 of 420 males $(30.2 \%)$ and 40 of 386 females $(9.9 \%)$ had G-6-PD deficiency. The predicted female incidence $(9.1 \%)$ according to the Hardy Weinberg equation was no analysed according to parental marriage patterns: $\%$ OF POPULATION \% OF DEFICIENT FEMALES A.Identical high risk B.Non-identical high risk C. High risk mother, low risk father

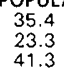

53.6
32.1

As expected, G.6.PD deficient females were more common in situations where both when the father incidene tather was isk a low rish olnic group, including Ash wenazi Jews in whom the incidence of G-5.PD deficiency is close to zero. We conclude that severe G-6-PD infrequently in females even if the father is not of a high risk family background. Female infants in high risk populations should be included in screening programs for G-6-PD deficiency for the prediction and evaluation of neonatal hyperbilirubinemia, regardless of paternal status.

\section{$\triangle 106$}

INFLUENCE OF PARITY ON MOTHER-TO-CHILD TRANSMISSION OF HIV Christian Kind* and the Pediatric AIDS Group of Switzerland Division of Neonatology, Kantonsspital, St.Gallen, Switzerland Background: Risk factors for mother-to-child transmission of HIV are still incompletely defined. An effect of parity has hitherto not been described.

Design: In a national prospective study, 316 children of HIV-positive mothers were followed up for at least 6 months. Infection status was determined in 254 of them according to the criteria of the Working Group on Mother-to-Child Transmission of HTV (AIDS 1993, 7:1139-1148) or, in children under 15 months, by concordant (positive or negative) results of virus detection tests in at least 2 samples by at least 2 methods (PCR, culture, antigen after immune complex disruption). Transmission rate was $18.1 \%$. The effect of potential risk factors on transmission rate was analysed. Results: Univariate analysis showed an association between primiparity and increased transmission rate: odds ratio (OR) 2.2, 95\%-confidence interval (95\%-CI) 1.1-4.6, $p<0.05$. Logistic regression confirmed this association (adjusted OR 2.4) and showed, in addition, a negative association between transmission rate and elective cesarean section (OR $0.36,95 \%$-CI $0.13-0.97, \mathrm{p}<0.05$ ). The effect of primiparity was less pronounced in combination with elective cesarean section (OR 1.7) than with other delivery modes (OR 2.5, difference not significant). HIV-infected children older than wo years were less likely to get a younger sibling during the observation period than cheir uninfected counterparts ( 0 of 22 vs. 10 of $101, p<0.05$ by logrank test). Conclusions: Primiparous women appear to transmit HIV to their children at a higher rate. This could be explained by increased intrapartum transmission because of longe and more complicated labour in primiparas and/or by a self-selection of women with lower risk of transmission among those deciding to have additional children.

\section{$\triangle 105$}

GLUCOCORTICOID SENSITIVITY OF HUMAN NEONATAL LEUKOCYTES

Annemieke Kavelaars', Gerard H.A. Visser. Ben J.M. Zegers', Jitske Zijlstra", and Cobi J. Heijnen

"Dept. Immunology, University Children Hospital "Het WIlhelmina Kinderziekenhuis", and Dept. of Obstetrics and Gynaecology, University Hospital, Utrecht, The Netherlands

Giucocorticoids (GC) are widely used as immunosuppressive and antiinflammatory drugs. They are known to inhibit the proliferative response of leukocytes after activation with mitogen, possibly by interfering with the interleukin-2 (IL-2) mRNA synthesis. Apart from IL-2 mRNA, GC also inhibit the transcription of genes encoding for IL-1 and IL-6. Until now, the effects of GC have predominantly been studied in adults. Therefore we studied the in vitro effects of the GC dexamethasone on the proliferative capacity of T cells in 15 human newborns. Our data show that neonatal leukocytes are more sensitive for inhibition of the proliferative response by dexamethasone than adult cells (EC 50 neonatal cells: $10^{-9} \mathrm{M}$; adults $3.10^{-8} \mathrm{M}<0.011$. This difference in sensitivity is not related to differences in GC receptor expression in neonatal and adult cells. Dexamethasone inhibits the expression of the IL-2 receptor on neonatal cells to a larger extent than on adult cells $(70 \%$ versus $25 \%$ inhibition). However, this difference can not explain the difference in sensitivity between adult and neonatal cells. We show that the increased sensitivity of neonatal cells for GC is due to their diminished capacity to produce IL-2. Addition of interleukin-2 can restore the proliferative capacity after dexamethasone inhibition of neonatal cell proliferation.
107

MOTOR TESTS OF INTERHEMISPHERIC CONTROL AND COGNITIVE FUNCTION IN VERY PRETERM INFANTS AT EIGHT YEARS. Vincent Kirkbride, Jenny Baudin, Ann Lorek, Judith Meek, Juliet Penrice, Jan Townsend, Simon Roth, David Edwards, David McCormick, Osmund Reynolds, Ann Stewart, Dept of Paediatrics, University College London Medical School, London, UK.

We have previously reported that damage to the posterior corpus callosum might explain some of the subtle cognitive processing deficits found later in many preterm infants. To test this hypothesis we carried out motor tests of interhemispheric control in 197 consecutively examined very preterm infants $(<33$ weeks gestation) at eight years. In addition to the Kaufman Assessment Battery for Children (K-ABC) and other studies, two tests of mirrorwise(Tm) and alternate $(\mathrm{T} a)$ forearm diadochokinetic (pronation-supination) movements were performed. The alternate movement represents the most complicated form of interhemispheric control and reciprocal inhibition. The time difference between the two movements (Ta- Tm) is considered to give an indication of the efficiency of transfer of motor information between the hemispheres (particularly the posterior corpus callosum). There was a significant and linear relation between this time difference and the Kaufman Mental Processing Composite (K-MPC) score $(p<0.002$, DF 2 ). We conclude that the corpus callosum has an important role in cognitive development and posterior corpus callosum efficiency is related to cognitive outcome.

\title{
$\triangle 108$
}

SURFACTANT DOWNREGULATES HUMAN MONOCYTE AND NEUTROPHIL RESPONSES TO HYPEROXIA. Haresh M. Kirpalani, Peter Conlon, Manel Jordana. Dept. of

Pediatrics McMaster Univ., Canada.
Monocytes (M) and neutrophils (N) are implicated in hyperoxic lung Monocytes (M) and neutrophils (N) are implicated in hyperoxic lung
injury. We investigated effects of surfactant (ST) on $N$ and $M$ in hyperoxia (HO2). METHOOS: $N$ and $M$ from heal thy adults were plated at $1 \times 10^{6}-4 \times 10^{6} / \mathrm{ml}$. Cells were incubated with $(+\mathrm{ST})$ or without $(-\mathrm{ST})$ surfactant $(100 \mu \mathrm{g} / \mathrm{ml}$; Exosurf $)$ and exposed to either $\mathrm{HO}\left(95 \% \mathrm{O}_{2}+5 \%\right.$ $\mathrm{CO}_{2}$ ) or air, for 2 to 18 hours. Supernatants was examined for TNF- $\alpha$ and IL-8 (ELISA). Cells were examined for PMA stimulated oxygen Radical (OR) production by luminol dependent chemiluminescence. RESULTS: i) By 2 hours HO2 increased OR by $N$; ST reduced OR, both in a ir and $\mathrm{HO}_{02}$ ( 3 subjects). Peak OR (mV) as (Mean(SD).

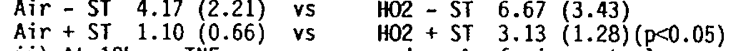

ii) At 18hrs: TNF $-\alpha$ was expressed as $\%$ of air control.

M:Air - ST HO2 - ST HO2 + ST (6 subjects)

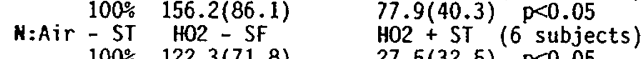
$\begin{array}{lll}100 \% & 122.3(71.8) & 27.5(32.5) \quad p<0.05\end{array}$

In $N$ and $M$, INF- $\alpha$ release was markedly diminished by $S T$.

iii) At $18 \mathrm{hrs}$ : IL-8 was expressed as $\%$ of air control.

N:Air - ST HO - ST HO + ST (6 subjects) $100 \% 92.9(46.3) \quad 68.5(48.4) \quad p>0.05$.

In N IL-8 release was not signficantly affected by ST.

CONCLUSIONS:ST significantly downregulates $T N F-\alpha$ and $O R$ release by 